\title{
An Evaluation of Deformation-Based Morphometry Applied to the Developing Human Brain and Detection of Volumetric Changes Associated with Preterm Birth
}

\author{
J.P. Boardman ${ }^{1}$, K. Bhatia ${ }^{2}$, S. Counsell ${ }^{3}$, J. Allsop ${ }^{3}$, O. Kapellou ${ }^{1}$, \\ M.A. Rutherford ${ }^{1,3}$, A.D. Edwards ${ }^{1}$, J.V. Hajnal ${ }^{1}$, and D. Rueckert ${ }^{2}$ \\ 1 Department of Paediatrics, Obstetrics and Gynaecology, Imperial College London \\ ${ }^{2}$ Visual Information Processing Group, Department of Computing, Imperial College London \\ ${ }^{3}$ Robert Steiner Magnetic Resonance Unit, Imaging Sciences Department, MRC Clinical \\ Sciences Centre, Imperial College London
}

\begin{abstract}
Deformation-based morphometry enables the automatic quantification of neuroanatomical differences by measuring regional shape and volume differences between a reference space and the population under investigation. In this paper we use deformation-based morphometric methods to study volumetric differences between preterm infants at term equivalent age and term born controls using high-resolution MR imaging, and we investigate the influence of the choice of reference image on results obtained. We constructed mean atlases of preterm infants at term equivalent age and term born infants using three different reference spaces, and used them to compare the brains of the two groups. A non-rigid registration algorithm was used to map all 3 atlases into a common coordinate system and volumetric differences were extracted. Our results demonstrate significant volume differences between preterm infants at term equivalent age and the control group in the ventricular system, the interhemispheric fissure anteriorly, and the globus pallidus and thalamus. Morphometric changes are consistent between all three maps of volume change and indicate that the results obtained using deformation-based morphometry are largely independent of the choice of the reference space.
\end{abstract}

\section{Introduction}

Preterm delivery affects 5\% of all deliveries and its consequences contribute to significant individual, medical and social problems. The principle morbidity among survivors is neurological, resulting from the profound effect of preterm birth on the developing brain: half of all infants born at less than 25 weeks have neurodevelopmental impairment at 30 months of age and in less immature infants neuropsychiatric problems are common in the teenage years [12]. This group of infants can be studied to evaluate the neuroimaging correlates of cognitive and behavioural impairments. Serial high resolution magnetic resonance (MR) brain imaging has shown that focal lesions are common around the time of birth among preterm infants but these can change or resolve with time; however subtle diffuse abnormalities of white matter and cortex are common at term equivalent age [3]. We have mapped logarithmic increases in the complexity of cortical folding, cerebral tissue volume and cortical surface area from 23 weeks gestation onwards, but 
in comparison to term born infants, preterm infants have reduced cortical surface area and complexity at term equivalent age [4]; reduced cortical development associated with white matter disease in preterm infants is also evidenced by others findings [5]. Cerebral structural abnormalities in association with cognitive deficits have been described in children and adolescents who were born preterm [67], and these differences are likely to arise in the neonatal period [8]. Although it is probable that diffuse abnormalities form the bases for subsequent impairment, the evolution and phenotype of preterm brain injury and the neural correlates of functional disorders are poorly characterised.

Computational morphometry has been used in a number of neurological [9 10,11] and neurodevelopmental [126.7] disorders to capture novel information about subtle neuroanatomical differences between patient groups. The technique relies on image registration to align multiple images before analysing for group differences. The developing brain offers challenges to morphometric techniques that use a low-dimnesional transformation to achieve alignment between the subjects and reference space because it is characterised by rapid increases in growth and complexity, the appearance of new structures, and evolving myelination (which independently alters the MR signal). We have used a deformation-based morphometric (DBM) approach [13]14], in which high-dimensional non-rigid registration is used to achieve a high degree of anatomical correspondence between an atlas (or a reference subject) and all other subjects in the group under investigation. Following registration, information about the anatomical differences between the atlas or reference subject and the population group can be extracted from the deformation fields. To characterise shape differences we use the Jacobian determinant of the deformations as a measure of regional tissue volume change [15,1411]. A common dilemma in deformation-based morphometry is the choice or construction of the atlas or reference space to which all subjects in the group are registered. While methods for computing an average anatomical model of the brain do exist [1617], it is not clear whether bias in the choice of atlas affects the results obtained using deformation-based morphometry. In this paper we use a high dimensional non-rigid registration algorithm [18] to examine morphometric differences between preterm and term born infants, and investigate the influence of the choice of atlas on results obtained.

\section{Methods}

\subsection{Subjects and Image Acquisition}

The MR images of 66 preterm infants (median 29.6, range 26-34 weeks post-menstrual age) were analysed at term equivalent age ( 38 to 42 weeks), together with those from 11 term control infants (median 39.6, range 38-42 weeks post-menstrual age). Ethical permission for this study was granted by the Hammersmith Hospital Research Ethics Committee and informed parental consent was obtained for each infant. Infants were sedated for the examination but none required mechanical ventilation at the time of MR imaging. Pulse oximetry, electrocardiographic and televisual monitoring were used throughout the examination which was attended by a paediatrician. For some analyses we defined a subgroup of 36 individuals with a post-menstrual age of less than 30 weeks. A 1.5 T Eclipse MR system (Philips Medical Systems, Cleveland, Ohio) was used to acquire high resolution $\mathrm{T} 1$ weighted images $\left(\mathrm{TR}=30 \mathrm{~ms}\right.$, $\mathrm{TE}=4.5 \mathrm{~ms}$, flip angle $=30^{\circ}$ ). In 
addition to conventional $\mathrm{T} 1$ and $\mathrm{T} 2$ weighted image acquisition, volume datasets were acquired in contiguous sagittal slices (in-plane matrix size $256 \times 256, \mathrm{FOV}=25 \mathrm{~cm}$ ) with a voxel size of $1.0 \times 1.0 \times 1.6 \mathrm{~mm}^{3}$.

\subsection{Non-rigid Registration}

In order to map the anatomy of each subject $\mathcal{S}$ into the anatomy of the atlas $\mathcal{R}$ it is necessary to employ non-rigid transformations such as elastic or fluid transformations. We have used a non-rigid registration algorithm which has been successfully applied successfully to a number of different registration tasks [18 19]. This algorithm uses a combined transformation $\mathbf{T}$ which consists of a global transformation and a local transformation. The global transformation describes the overall differences between the two subjects which only accounts for differences due to position, orientation and size of the anatomy and is represented by an affine transformation. The local transformation describes any local deformation required to match the anatomies of the subjects. We have chosen a free-form deformation (FFD) model based on B-splines which is a powerful tool for modelling 3D deformable objects. In essence FFDs deform an object by manipulating an underlying mesh of control points. The resulting deformation controls the shape of the $3 \mathrm{D}$ object and can be written as the 3D tensor product of the familiar 1D cubic B-splines,

$$
\mathbf{T}_{\text {local }}(\mathbf{x})=\sum_{l=0}^{3} \sum_{m=0}^{3} \sum_{n=0}^{3} B_{l}(u) B_{m}(v) B_{n}(w) \mathbf{c}_{i+l, j+m, k+n}
$$

where $\mathbf{c}$ denotes the control points which parameterise the transformation. The optimal transformation is found by minimising a cost function associated with the global transformation parameters as well as the local transformation parameters. The cost function comprises two competing goals: the first term represents the cost associated with the voxel-based similarity measure, in this case normalised mutual information [20], while the second term corresponds to a regularisation term which constrains the transformation to be smooth [18]. The resulting transformation $\mathbf{T}$ maps each point in the anatomy of the reference subject $\mathcal{R}$ to the corresponding point in the anatomy of the subject $\mathcal{S}$.

\subsection{Deformation-Based Morphometry}

To compare the neuroanatomical phenotpyes of the two groups we selected MR images of three infants born at term which formed three different reference spaces to which T1 weighted volume datasets from 66 preterm at term equivalent age and 11 term born controls were registered. In the first step, we calculated the global transformation between the subjects and the atlas correcting for scaling, skew, rotation and translation. We then applied the non-rigid registration algorithm using a multi-resolution scheme with control spacing of $20 \mathrm{~mm}, 10 \mathrm{~mm}, 5 \mathrm{~mm}$ and $2.5 \mathrm{~mm}$. The resulting control point mesh defines a $C^{2}$ continuous and analytic representation of the deformation field which describes the point-wise 3D displacement vectors that are required to warp each dataset to the reference image. The registrations between subjects and reference subject have been carried out for all three reference subjects and all registered images were checked for artefacts and 
accurate anatomical localisation by visual inspection. In all cases registration achieved a visually plausible alignment of anatomical structures.

\subsection{Data Analysis}

To calculate regional volume changes the determinant of the Jacobian of the deformation field is used to quantify differences between registered images and reference. The determinant of the Jacobian for any given location in the reference coordinate system for each individual provides an estimate of the point-wise volume change of that individual with respect to the atlas. Values above 1 indicate tissue expansion, values below 1 indicate tissue contraction, values below 0 indicate folding and infinite values indicate tearing. To express regional volume differences between two groups we have calculated the effect size [11.921]:

$$
\epsilon(\mathbf{x})=\frac{\mu_{A}(\mathbf{x})-\mu_{B}(\mathbf{x})}{\sigma_{A \cup B}(\mathbf{x})}
$$

Here $\mu_{A}(\mathbf{x})$ and $\mu_{A}(\mathbf{x})$ denote the mean Jacobian value at $\mathbf{x}$ for group A and $\mathrm{B}$ while $\sigma_{A \cup B}(\mathbf{x})$ denotes the standard deviation of the Jacobian values at $\mathbf{x}$ for the pooled group.

To evaluate the consistency of the deformation-based approach, we calculated volume change maps between each subject $\mathbf{S}$ and all three reference subjects, $\mathcal{R}_{\# 1}, \mathcal{R}_{\# 2}$ and $\mathcal{R}_{\# 3}$. We also registered $\mathcal{R}_{\# 2}$ and $\mathcal{R}_{\# 3}$ to $\mathcal{R}_{\# 1}$ which allows us to transform each volume change map into the coordinate system of $\mathcal{R}_{\# 1}$. In order to demonstrate regional consistency we segmented the lateral ventricular system and a region within the deep grey nuclei of $\mathcal{R}_{\# 1}$ and propogated these segmentations to $\mathcal{R}_{\# 2}$ and $\mathcal{R}_{\# 3}$. The mean volume change of the subjects to these regions in $\mathcal{R}_{\# 2}$ and $\mathcal{R}_{\# 3}$ was scaled by the Jacbian determinant of the transformation of $\mathcal{R}_{\# 2}$ and $\mathcal{R}_{\# 3}$ to $\mathcal{R}_{\# 1}$ respectively. If the registrations had no associated errors, the volume change maps would be identical regardless of the choice of the reference subject.

\section{Results}

Figure 1 (a) shows one of the reference subjects (normal infant at term), (b) shows an intensity atlas produced by averaging the brains of 66 preterm at term equivalent age infants using affine registration to the reference subject and (c) shows an average intensity atlas produced by averaging the brains of 66 preterm at term equivalent age infants using non-rigid registration to the reference subject. A quantitative comparison of regional volumetric differences between the preterm at term equivalent age group and the term born infants is shown in table 1 There is increased volume in the lateral ventricles and there is volume reduction in the deep gray nuclei in the preterm at term equivalent age group compared to the term born group. Table 1 also shows the effect of using different reference subjects as the standard space in which to compare the volume changes. Both the effect size and the volume change show a large degree of consistency regardless of the reference subject used. A qualitative comparison of the effect size is shown in Figure 2: the isolines represent regions of equal effect size and in the top row (a)-(c) the tissue contained within the isoline, a region within deep grey matter encompassing globus pallidus and thalamus, is more contracted in the preterm 
at term equivalent age group compared to the infants born at term. The isolines in row (d)-(i) show areas of relative tissue expansion in the preterm group compared to the term infants. These areas are localised to the posterior horns of the lateral ventricular system, the cerebrospinal fluid spaces, and the anterior part of the interhemispheric fissure. These distributions of volume change were replicated using three different reference images.

Table 1. Comparison of the effect size and the volume change measurements in the ventricles and deep grey nuclei for each reference subject, with values of volume change to $\mathcal{R}_{\# 2}$ and $\mathcal{R}_{\# 3}$ scaled to the coordinate system of $\mathcal{R}_{\# 1}$. These values show that volume changes are consistent irrespective of the reference space used.

\begin{tabular}{|c|c|c|c|c|c|c|c|}
\hline \multirow{2}{*}{ ROI } & \multirow{2}{*}{ Subject group } & \multicolumn{2}{|c|}{ Effect size } & \multicolumn{3}{c|}{ Volume change } \\
\cline { 3 - 7 } & & $\mathcal{R}_{\# 1}$ & $\mathcal{R}_{\# 2}$ & $\mathcal{R}_{\# 3}$ & $\mathcal{R}_{\# 1}$ & $\mathcal{R}_{\# 2}$ & $\mathcal{R}_{\# 3}$ \\
\hline \multirow{2}{*}{ Basal ganglia } & pre-term $>30$ weeks & -0.89 & -0.76 & -0.87 & $79 \%$ & $84 \%$ & $75 \%$ \\
\cline { 2 - 7 } & pre-term $<30$ weeks & -1.04 & -0.84 & -0.99 & $72 \%$ & $79 \%$ & $74 \%$ \\
\hline \multirow{2}{*}{ Ventricles } & pre-term $>30$ weeks & 0.47 & 0.46 & 0.41 & $123 \%$ & $129 \%$ & $122 \%$ \\
\cline { 2 - 7 } & pre-term $<30$ weeks & 0.75 & 0.70 & 0.68 & $127 \%$ & $131 \%$ & $127 \%$ \\
\hline
\end{tabular}

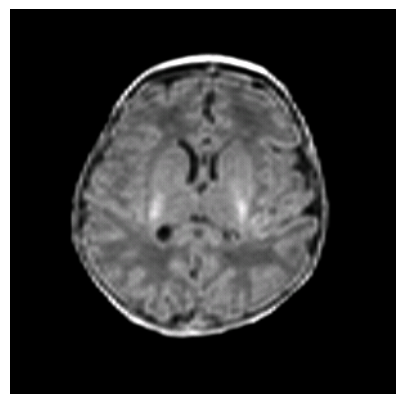

(a)

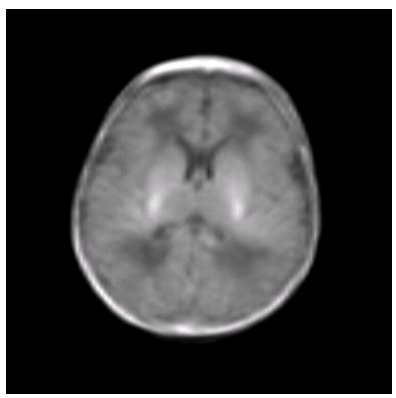

(b)

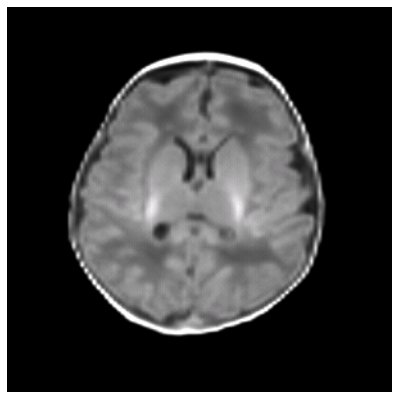

(c)

Fig. 1. (a) shows one of the reference subjects (normal infant at term), (b) shows an average intensity atlas produced by averaging the brains of 66 pre-term at term equivalent age infants using affine registration to the reference subject and (c) shows an average intensity atlas produced by averaging the brains of the same preterm at term equivalent age group using non-rigid registration to the reference subject.

\section{Discussion}

We have used a high dimensional non-rigid registration algorithm to study a large dataset of neonatal MR brain images, and have identified morphometric changes associated with preterm birth. We have observed enlargement of the posterior horns of the lateral ventricles, widening of the interhemispheric fissure anteriorly, and localised tissue contraction in the globus pallidus and thalamus. Ventriculomegaly and widening of the anterior interhemispheric fissure have both been reported in preterm infants at term equivalent age, but these changes have not been quantified [3]. In separate studies using diffusion 


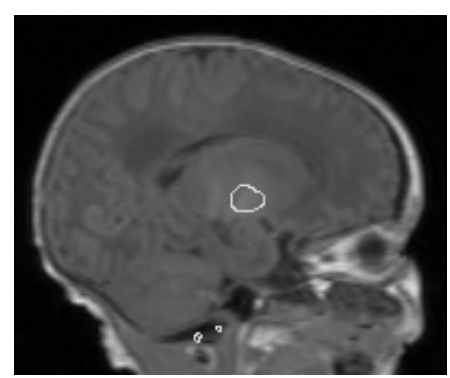

(a)

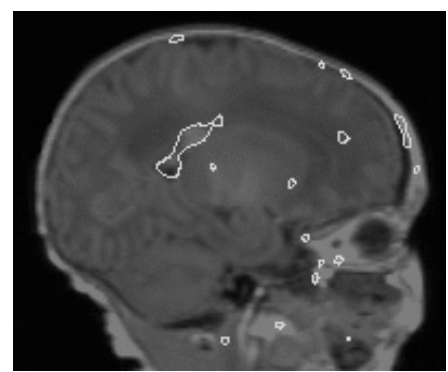

(d)

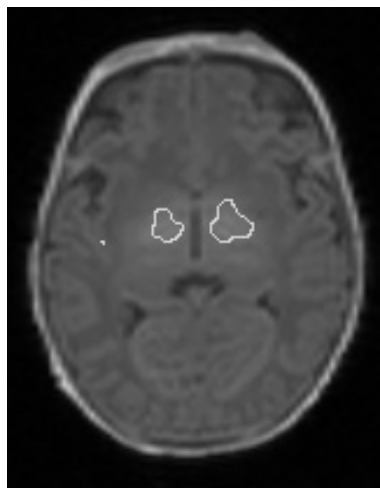

(b)

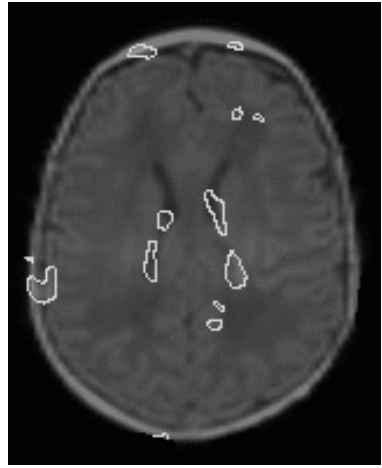

(e)

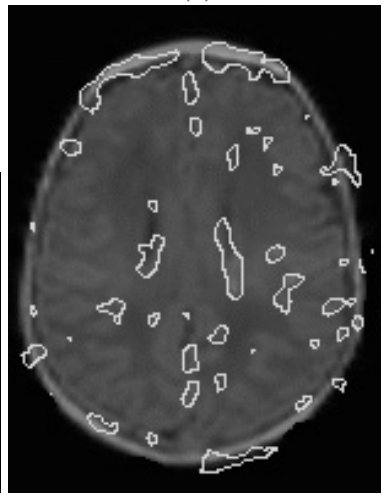

(h)

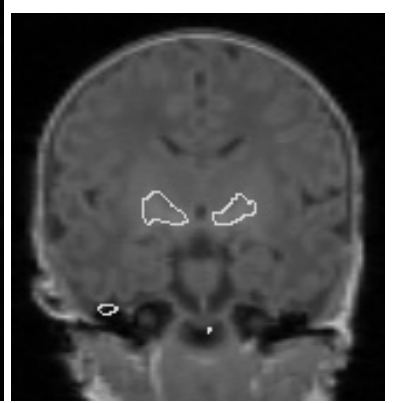

(c)

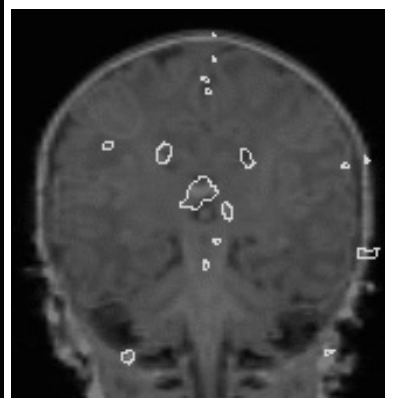

(f)

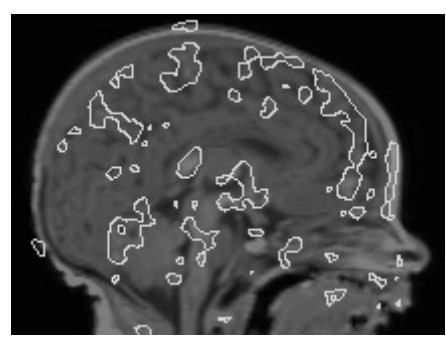

(g)

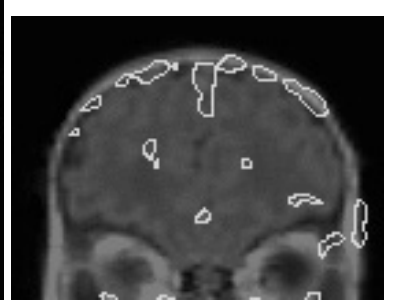

(i)

Fig. 2. This figure shows example sagittal, axial, and coronal slices illustrating the spatial distribution of the effect size of the Jacobian determinant in both groups superimposed on a reference image. The isolines represent regions of equal effect size and in the top row (a)-(c) the tissue contained within the isoline (effect size $\epsilon<-1.3$ ), a region within the deep grey nuclei encompassing globus pallidus and thalamus, is more contracted in the preterm at term equivalent age group compared to the term controls. The isolines in figures (d)-(i) show areas of relative tissue expansion in the preterm group compared to the term infants. These areas are localised to the lateral ventricular system (effect size $\epsilon>0.9$ ) and the interhemispheric fissure anteriorly, and cerebrospinal fluid spaces around the frontal lobes (effect size $\epsilon>0.7$ ). These tissue distributions of morphometric change were replicated using three different reference images. 
weighted MR imaging we have found that apparent diffusion coefficient (ADC) values, which provide an objective measure of tissue connectivity, are higher in frontal lobe white matter (adjacent to anterior interhemispheric fissure) than in other brain regions [22] suggesting an increased susceptibilty of frontal white matter to tissue injury. In future analyses this tool could be used to explore the relationship between ADC values and morphometric change.

Tissue contraction in the deep grey nuclei was unexpected and highlights the utility of this technique in surveying the brain for volumetric changes without prior hypotheses. There are two possible explanations for tissue contraction in this region: central grey matter may be preferentially injured by factors present in the extrauterine environment such as hypoxia or inadequate nutrition; or volume changes could be associated with myelinating white matter tracts of the posterior limb of the internal capsule as they traverse central grey matter. There is wider variation in the degree of myelination of white matter tracts in this region among term born infants in contrast with preterm infants among whom myelination takes place prematurely in this tract. The volumetric effect of variability in myelination between the two groups will be explored in subsequent analyses by increasing the number of subjects in the term control group.

This study has demonstrated the utility and consistency of a non-rigid image registration algorithm in defining the morphometric phenotype of preterm brain injury, and shown that these findings are largely independent of the choice of reference anatomy used. Other metrics of shape change could be extracted and statistical analyses applied to further characterise these changes; specifically, studying infants longitudinally throughout this period of brain development, and exploring datasets for associated locations of volume change will further knowledge of the neuroanatomic sequence of injury. Defining the phenotype of preterm brain injury will enable relationships with collateral clinical, imaging, biochemical or genetic data to be explored. Therefore the technique provides an opportunity to relate structure to functional outcome, and offers a quantitative tool for testing hypotheses concerning the aetiology of injury, and the efficacy of preventative strategies.

Acknowledgements. Jo Hajnal, Joanna Allsop, Mary Rutherford, Serena Counsell, David Edwards and James Boardman were funded by the Medical Research Council. Kanwal Bhatia was funded by EPSRC grant GR/S08916/01. The Robert Steiner MR Unit is supported by Philips Medical Systems.

\section{References}

1. N. S. Wood, N. Marlow, et al. Neurologic and developmental disability after extremely preterm birth. New England Journal of Medicine, 343(6): 429-430, 2000.

2. N. Botting, A. Powls, et al. Attention deficit hyperactivity disorders and other psychiatric outcomes in very low birthweight children at 12 years. J Child Psychol Psychiatry, pages 931-941, 1997.

3. E. F. Maalouf, P. J. Duggan, et al. Magnetic resonance imaging of the brain in a cohort of extremely preterm infants. Journal of Pediatrics, 135(3): 351-357, 1999.

4. M. Ajayi-Obe, N. Saeed, et al. Reduced development of cerebral cortex in extremely preterm infants. Lancet, 356(9236): 1162-3, 2000. 
5. T. E. Inder, P. S. Huppi, et al. Periventricular white matter injury in the premature infant followed by reduced cerebral cortical gray matter voliume at term. Annals of Neurology, pages 755-760, 1999.

6. E. B. Isaacs, C. J. Edmonds, et al. Calculation difficulties in children of very low birthweight - a neural correlate. Brain, 124: 1701-1707, 2001.

7. B. S. Peterson, B. Vohr, et al. Regional brain volume abnormalities and long-term cognitive outcome in pre-term infants. JAMA, pages 1939-1947, 2000.

8. B. S. Peterson, A. W. Anderson, et al. Regional brain volumes and their later neurodevelopmental correlates in term and preterm infants. Pediatrics, 111: 939-948, 2003.

9. C. Davatzikos, M. Vaillant, et al. A computerized approach for morphological analysis of the corpus callosum. Journal of Computer Assisted Tomography, 20: 88-97, 1996.

10. J. G. Cseransky, S. Joshi, et al. Hippocampal morphometry in schizophrenia by highdimensional brain mapping. In Proc. Natl. Acad. Sci. USA, volume 95, pages 11406-11411, 1998.

11. C. Studholme, V. Cardenas, et al. Detecting spatially consitent structural differences in alzheimer's and fronto temporal dementia using deformation morphometry. In Fourth Int. Conf. on Medical Image Computing and Computer-Assisted Intervention (MICCAI '01), pages 41-48, 2001.

12. P. M. Thompson, J. N. Giedd, et al. Growth patterns in the developing brain detected by continuum mechanical tensor maps. Nature, 404: 190-193, March 2000.

13. J. Ashburner, C. Hutton, et al. Identifying global anatomical differences: Deformation-based morphometry. Human Brain Mapping, 6: 638-57, 1998.

14. C. Gaser, I. Nenadoc, et al. Deformation-based morphometry and its relation to conventional volumetry of brain lateral ventricles in MRI. NeuroImage, 13: 1140-1145, 2001.

15. J.-P. Thirion and G. Calmon. Deformation analysis to detect and quantify active lesions in three-dimensional medical image sequences. IEEE Transactions on Medical Imaging, 18(5): 429-441, 1999.

16. A. Guimond, J. Meunier, and J.-P. Thirion. Average brain models. Computer Vision and Image Understanding, 77: 192-210, 2000.

17. D. Rueckert, A. F. Frangi, and J. A. Schnabel. Automatic construction of 3D statistical deformation models using non-rigid registration. In Fourth Int. Conf. on Medical Image Computing and Computer-Assisted Intervention (MICCAI '01).

18. D. Rueckert, L. I. Sonoda, et al. Non-rigid registration using free-form deformations: Application to breast MR images. IEEE Transactions on Medical Imaging, 18(8): 712-721, 1999.

19. J. A. Schnabel, D. Rueckert, et al. A generic framework for non-rigid registration based on non-uniform multi-level free-form deformations. In Fourth Int. Conf. on Medical Image Computing and Computer-Assisted Intervention (MICCAI '01), pages 573-581.

20. C. Studholme, D. L. G. Hill, and D. J. Hawkes. An overlap invariant entropy measure of 3D medical image alignment. Pattern Recognition, 32(1): 71-86, 1998.

21. A. Machado and J. Gee. Atlas warping for brain morphometry. In Proc. SPIE Medical Imaging 1999: Image Processing, 1998.

22. S.J. Counsell, J.M. Allsop, et al. Diffusion-weighted imaging of the brain in preterm infants with focal and diffuse white matter abnormality. Pediatrics, 112(1): 1-7, 2003. 